\title{
Drug Resistance in Cancer Therapy and the Role of Epigenetics
}

\author{
Takeshi Asano
}

Department of Pediatrics, Nippon Medical School, Tokyo, Japan

\begin{abstract}
Effective leukemia treatment is seriously hampered by drug resistance, and the potential role of epigenetic mechanisms in cancer drug resistance has recently been investigated. With conventional anticancer drugs, including alkylating drugs, anti-metabolite drugs, topoisomerase inhibitors, and microtubule inhibitors - which have been available for half a century-drug resistance often develops because of decreased expression of target enzymes, in conjunction with increased expression of drug export pumps. Alterations of target gene expression and increased export pump function might be caused by epigenetic changes, such as alterations in methylation status, as well as by changes in histone acetylation status. In addition, newly developed anticancer drugs, including small-molecule drugs, such as kinase inhibitors, antibody drugs, and immune modulatory drugs, also resulted in development of drug resistance within 1 year, although these drugs showed significant effectiveness for patients resistant to conventional anticancer drugs. The resistant cells exhibited increased expression of bypass pathways, activation of downstream cascades, decreased expression of antigens of tumor cells, increased DNA repair activity, and increased expression of drug export pumps, which also suggests the presence of epigenetic changes. This article reviews drug resistance in cancer therapy and the possible roles of epigenetic mechanisms. (J Nippon Med Sch 2020; 87: 244-251)
\end{abstract}

Key words: leukemia, drug resistance, epigenetics

\section{Introduction}

Drug resistance remains a serious problem in cancer therapy, and outcomes are substantially worse for children with cancer cells exhibiting in vitro resistance to anticancer agents than for children with drug-sensitive cancer cells ${ }^{1}$. Gene expression patterns in cancer cells that eventually acquire resistance before the start of treatment are important because drug-resistant subpopulations grow selectively as treatment progresses. Nevertheless, the mechanisms by which cells acquire drug resistance are unclear. Many signaling pathways and genes that may affect the response of cancer cells to therapy have been identified. Because numerous factors are involved in the acquisition of drug resistance, the "one gene: one outcome" hypothesis cannot adequately explain acquired resistance in cancer ${ }^{2}$. Thus, multiple mechanisms and multiple genes, rather than a single pathway or gene, likely mediate acquired resistance.

Aberrant methylation has a potent role in tumorigene- sis, and genome-wide hypomethylation and regional hypermethylation of tumor suppressor gene promoters are characteristic hallmarks of many cancers ${ }^{3}$. DNA methylation occurs in eukaryote DNA at CpG sites, usually enriched in gene promoters. Increasing evidence indicates that epigenetic changes are crucial in the acquisition of drug resistance and that changes in gene expression occur after chemotherapy without gene mutations ${ }^{4-6}$. Using drug-resistant cell lines and differential methylation hybridization, Wei et al. found many differences in CpG island methylation and epigenetic regulation after drug treatment ${ }^{7}$.

Histones also control gene expression by modulating the structure of chromatin and accessibility of regulatory DNA sequences to transcriptional activators and repressors $^{8,9}$. Acetylation of histone increases gene expression by relaxing chromatin structure, thereby allowing access of transcription factors to DNA ${ }^{5}$.

This article reviews drug resistance to cancer therapy

Correspondence to Takeshi Asano, M.D., Department of Pediatrics, Nippon Medical School Chiba Hokusoh Hospital, 1715

Kamagari, Inzai, Chiba 270-1694, Japan

E-mail: july1364@nms.ac.jp

https://doi.org/10.1272/jnms.JNMS.2020_87-508

Journal Website (https://www.nms.ac.jp/sh/jnms/) 
and the possible roles of epigenetic mechanisms.

\section{Conventional Anticancer Drugs}

Conventional anticancer drugs include alkylating drugs, antimetabolite drugs, topoisomerase inhibitors, and microtubule inhibitors, all of which have been available for half a century. In the 1990s, many studies investigated drug resistance, especially multiple-drug resistance ${ }^{10}$. The mechanisms of anticancer drug resistance were generally accepted to be increased drug metabolism, decreased drug uptake, decreased expression of target molecules, and mutation of target molecules.

\section{Alkylating Drugs}

1) Mechanisms of drug action

Alkylating agents bind alkyl bases of DNA in tumor cells and make a covalent conjunction between drugs (dacarbazine, temozolomide) and one alkyl base, or between drugs (cyclophosphamide, melphalan) and two alkyl bases. These modified alkylated bases are not able to repair because of the absence of a DNA repair mechanism in leukemic cells. This leads to apoptosis in leukemic cells.

2) Mechanisms of resistance to alkylating drugs

The reported mechanisms of resistance to alkylating drugs are (1) decreased drug transfer into cells, (2) inactivation of glutathione as a detoxifying enzyme, (3) enhancement of O6-alkylguanine methyl converting enzyme (MGMT), (4) enhancement of nucleotide excision repair as a DNA repair function, and (5) deficits in mismatch repair ${ }^{11}$.

(1) Decreased uptake into tumor cells: Decreased drug uptake into cancer cells results from increased expression and function of export pumps, which include ATPbinding cassette $(\mathrm{ABC})$ transporters. Using ATP, these transporters export anticancer drugs outside cancer cells. To date, 49 proteins (transporters) have been identified as $\mathrm{ABC}$ transporters ${ }^{10}$. The present author found no mutations in the promoter area of $\mathrm{ABC}$ transporter genes in drug-resistant cancer cell lines with alkylating agents ${ }^{12}$. Currently, increased expression of ABC transporter genes is believed to develop through epigenetic regulation ${ }^{13,14}$.

(2) Increased detoxification in tumor cells caused by increased expression of glutathione transferases: Increased detoxification is generally caused by increased expression and function of glutathione transferases. Increased expression of these genes might be related to epigenetic mechanisms ${ }^{15}$.

(3) Increased expression of MGMT: MGMT is a DNA repair enzyme, and low MGMT expression increases drug sensitivity in cancer cells. Because lower expression is related to methylation of the promoter region of MGMT gene ${ }^{16}$, hypomethylation might occur in leukemia cells resistant to alkylating drugs.

(4) Enhancement of nucleotide excision repair as a DNA repair function: Alkylating agents damage tumor cells by covalently linking the alkyl group to DNA. Enhancement of the repair mechanism leads to resistance to alkylating agents. These alterations in the expression of DNA repair enzymes might be caused by epigenetic modulations.

(5) Lack of mismatch repair: The alkylating agent forms a base pair with thymine by alkylation at the $\mathrm{O} 6$ position and causes mismatch formation. In resistant cells, apoptosis is not induced by eliminating the mismatch repair, and resistance against alkylating agents develops.

\section{Antimetabolite Drugs}

This category of drugs includes pyrimidine analogs (cytosine arabinoside [Ara-C], enocitarabine [BHAC], gemcitabine), purine analogs (fludarabine, 6mercaputopurine, nelarabine, clofarabine), and anti-folic acid drugs (methotrexate).

1) Drug actions of pyrimidine nucleoside analogs

The processes involved in the drug actions of pyrimidine nucleoside analogs (especially Ara-C) are (1) uptake to cancer cells by nucleoside transporter (human equilibrative nucleoside transporter 1 [hENT1]); (2) phosphorylation by deoxycytidine kinase; and (3) binding of DNA and blocking elongation of DNA chains and induction of apoptosis ${ }^{17}$.

2) Mechanisms of resistance to Ara-C

(1) Decreased expression of nucleoside transporters: Decreases in expression and function of nucleoside transporters are related to resistance to pyrimidine analogs. Decreased expression might be caused by increased methylation of the promoter region of the hENT1 gene or decreased acetylation of the hENT1 gene ${ }^{17}$.

(2) Increased expression of cytidine deaminase and decreased activity of Ara-C: Ara-C is inactivated by the action of cytidine deaminase. When cytidine deaminase activity increases, drug resistance is acquired because Ara$C$ activity is reduced ${ }^{18}$.

(3) Decreased expression or mutation of deoxycytidine kinase and decreased phosphorylation of Ara-C: Intracellular Ara-C is phosphorylated to Ara-CMP by the action of deoxycytidine kinase, and further phosphorylation of Ara-CMP results in active Ara-CTP. Active Ara-CTP becomes a substrate for DNA polymerase and is translo- 
cated into the nucleus, which inhibits elongation of the DNA chain and ultimately induces apoptosis. Reduced deoxycytidine deaminase activity causes drug resistance ${ }^{19}$.

(4) Decreased expression of deoxycytidine monophosphate (dCMP) deaminase and increased production of dCTP: Ara-CMP is phosphorylated and becomes an active form, Ara-CTP, which causes cell damage. However, a decrease in dCMP deaminase activity increases conversion of Ara-CMP to Ara-UMP, resulting in resistance. Furthermore, dCTP competitively inhibits DNA polymerase with Ara-C, so that increasing dCTP results in resistance to Ara-C $\mathrm{C}^{20}$.

(5) Increased expression of anti-apoptosis protein: Increases in Bcl-2 expression and function lead to antiapoptotic action in resistant cells ${ }^{21}$.

(6) Increased expression of $3^{\prime} \rightarrow 5^{\prime}$ endonuclease: Ara-C is translocated to the nucleus, and $3^{\prime} \rightarrow 5^{\prime}$ endonuclease leads to removal of DNA that is integrated with pyrimidine analogs, such as Ara-C. Thus, increased endonuclease activity causes Ara-C resistance.

3) Mechanisms of drug action of purine nucleotide analogs

The drug actions of purine nucleotide analogs (fludarabine, 6-mercaputopurine, nelarabine, clofarabine) are detailed below.

(1) Uptake into tumor cells by nucleotide transporter: The purine nucleotide analog is transferred to the cell via a nucleotide transporter, such as Ara-C.

(2) Phosphorylation by deoxycytidine kinase (dCK): In the cell, purine nucleotide analogs are phosphorylated by deoxycytidine kinase ( $\mathrm{dCK}$ ) and become triphosphates, and purine nucleotide analogs become their active forms.

(3) Integration into DNA and inhibition of DNA synthesis: The triphosphorylated purine nucleotide analogs translocate into the nucleus and are incorporated into DNA, thereby inhibiting DNA synthesis.

4) Mechanisms of resistance to purine analogs ${ }^{5}$

(1) Decreased transport (hENT1) into tumor cells: hENT1 is a necessary pump for transporting a drug into a cell. Decreased uptake into cells causes drug resistance.

(2) Decreased phosphorylation by decreased expression of the deoxycytidine kinase (dCK) gene: Purine nucleotide analogs taken into cells are phosphorylated by dCK. If the function of $\mathrm{dCK}$ is reduced, purine nucleotide analogs are phosphorylated and do not become their active forms, thereby causing drug resistance. Yamanishi et al. reported that resistance to clofarabine was caused by decreased expression of dCK gene, which resulted from decreased deacetylation of histone in the promoter region of $\mathrm{dCK}$ and a concomitant decline in function, thus causing drug resistance, as summarized in Table $\mathbf{1}^{5}$.

5) Drug action of 6-mercaputopurine (6-MP)

6-MP is a key drug in childhood acute lymphoblastic leukemia. 6-MP is transferred into the cell and converted to thioinosine monophosphate (TIMP). It inhibits biosynthesis of adenylic acid and guanylic acid and has antitumor activity ${ }^{22}$.

6) Mechanisms of resistance to 6-MP

The reported mechanisms of resistance to 6-MP are oxidation to the inactivated form by xanthine oxidase and methylation by thiopurine methyltransferase (TPMT), which has a genetic polymorphism with reduced activity. A correlation between gene polymorphism and 6-MP metabolic activity has been reported, and mutant-type TPMT results in more severe bone marrow suppression than does wild-type $\mathrm{TPMT}^{22}$.

7) Drug actions of anti-folic acid drugs (methotrexate)

Folic acid is essential for cell survival. Because de novo folic acid biosynthesis is not possible in mammals, folate must be obtained from outside of cells before being used in DNA synthesis. Methotrexate (MTX) is an enzyme that inhibits the action of dihydrofolate reductase (DHFR), which reduces folic acid to the active folic acid required for nucleic acid synthesis. MTX inhibits thymidylate and purine synthesis and suppresses cell growth.

8) Mechanisms of resistance to MTX

The reported mechanisms of resistance to MTX are (1) decreased cellular uptake of folic acid, (2) increased expression of thymidylate synthase (TS), (3) increased copy number of DHFR gene or mutation, and (4) decreased polyglutamine synthesis by decreased folypolyglutamate synthase (FPGS) activity ${ }^{23,24}$.

(1) Decreased cellular uptake of folic acid: MTX has a structure similar to that of folic acid and is actively transported into cells by reduced folate carrier (RFC). Decreased RFC activity reduces MTX uptake into cells.

(2) Increased expression of TS: DNA is synthesized by means of the thymidine kinase pathway. MTX is converted to the polyglutamine type, accumulates in cells, and binds DHFR. This binding inhibits nucleic acid synthesis and causes apoptosis.

(3) Increased copy number or gene mutation of DHFR: An increased copy number or mutation of DHFR prevents depletion of reduced folic acid and prevents leukemic cell death.

(4) Decreased polyglutamine synthesis by decreased FPGS activity: Decreased FPGS activity reduces polyglutamine synthesis and inhibits accumulation of the poly- 
Table 1
1) Drug actions of 6-mercaptopurine
(1) Transported into tumor cells and becomes thioinosine monophosphate (TIMP)
(2) Inhibition of synthesis of adenyl acid and guanyl acid.
2) Mechanisms of resistance to 6-mercaptopurine
(1) Inactivation by xanthine kinase
(2) Decreased expression of thiopurine methyltransferase\#
(3) Decreased expression of thiopurine methyltransferase (TPMT) by polymorphism
3) Drug actions of anti-folic acid drugs (methotrexate)
(1) Mammals cannot synthesize folic acid.
(2) Folic acid needs to be imported actively into cells by reduced folate carrier (RFC) and is used for DNA synthesis.
(3) Methotrexate is actively transported into tumor cells by the same reduced folate carrier (RFC).
(4) Methotrexate binds to dihydrofolate reductase by folypolyglutamate synthetase.
(5) Methotrexate inhibits deoxidation of the oxidative type of folic acid and leads to decreased deoxidation of folic acid.
(6) Methotrexate inhibits DNA synthesis along with thymidylate synthase and causes cell death.
4) Mechanisms of resistance to methotrexate
(1) Decreased expression of reduced folate carrier (RFC), which decreases uptake of folic acid.
(2) Increased expression of thymidylate synthase
(3) Increased copy number of the dihydrofolate reductase (DHFR) gene and/or mutation of the dihydrofolate reductase (DHFR) gene
(4) Inhibit polyglutamine oxidation by decreased expression of folypolyglutamate synthetase\#\#

\begin{abstract}
\#Decreased expression of thiopurine methyltransferase (TPMT) might be caused by increased methylation of the promoter region of the TPMT gene or decreased acetylation of the TPMT gene.

\#\#Decreased expression of reduced folate carrier, increased expression of thymidylate synthase, and decreased expression of folypolyglutamate synthetase might be caused by epigenetic mechanisms.
\end{abstract}

glutamine type of MTX.

\section{Topoisomerase Inhibitors}

1) Mechanisms of drug action

Topoisomerase is a key enzyme in the duplication and transcription of single-stranded and double-stranded DNA. Topoisomerase I cuts and ligates single-stranded DNA; camptothecin inhibits topoisomerase I. Topoisomerase II cuts and ligates double-stranded DNA, and inhibitors of topoisomerase II include etoposide and anthracycline ${ }^{4}$.

2) Mechanisms of resistance to topoisomerase inhibitors

(1) Increased expression of export pumps: $A B C$ transporter, P-glycoprotein (ABCB1), and breast cancer resistance protein (BCPR): Increased expression of $A B C$ transporters in drug-resistant cancer cells was not associated with mutations in the promoter area of these genes. This suggests that the mechanism of increased expression of $\mathrm{ABC}$ transporters might be related to epigenetics. ${ }^{4}$. However, MX2-a morpholino anthracycline derivative with highly lipophilic properties-did not increase expression of $\mathrm{ABC}$ transporters.

(2) Mutation of binding sites of topoisomerase: Mutations of the binding sites of topoisomerase result in fail- ure of the drug to interact with the enzyme in established cell lines. Although mutations have been reported in in vitro cell lines, mutations are rare in clinical specimens from patients with drug-resistant cancers ${ }^{4}$.

(3) Increased expression of DNA repair enzymes: DNA repair enzyme activity is greater in damaged cancer cells, which improves their survival.

(4) Decreased expression of topoisomerase II or mutation of topoisomerase II: Decreased expression of the topoisomerase II gene is frequently observed in topoisomerase II-resistant cells, and the author found that decreased expression was caused by increased methylation of promoter regions of the topoisomerase II $\alpha$ gene $e^{4}$. However, no mutations were observed in the topoisomerase II $\alpha$ gene or promoter in leukemia cells resistant to topoisomerase II $\alpha$ inhibitors ${ }^{4}$.

(5) Increased expression of glutathione reductase: Metabolism of camptothecin, an inhibitor of topoisomerase I, requires glucuronate conjugation. Increased expression of glutathione S-transferase increases glucuronate conjugation and detoxification.

\section{Anthracycline}

1) Mechanisms of drug action

The antitumor effect occurs by inhibiting DNA and 
RNA synthesis, DNA polymerase, and RNA polymerase, and by insertion of topoisomerase II between DNA base pairs of tumor cells ${ }^{25}$.

2) Mechanisms of resistance to anthracycline ${ }^{10}$

(1) Emerging multidrug-resistant proteins: Pglycoprotein, multidrug resistance-associated protein, lung resistance-related protein: Increased expression of multidrug-resistance proteins by enhanced expression of P-glycoprotein, multidrug-resistance protein multidrug resistance-associated protein, and lung resistance-related protein, among others, enhances the function of excreting anticancer drugs from leukemia cells and induces anthracycline drug resistance.

(2) Decreased topoisomerase activity: Decreased topoisomerase gene expression and activity are frequently observed in anthracycline-resistant tumor cells and in conjunction with decreased expression related to epigenetic mechanisms ${ }^{4}$. Mutations of the topoisomerase gene have been observed in resistant cell lines but are rare in human samples ${ }^{26}$.

(3) Increased detoxification: Increased expression of glutathione S-transferase and increased TS activity have been observed in anthracycline-resistant cells. Alteration of gene expression might be related to epigenetic mechanisms ${ }^{27}$.

\section{Microtubule Inhibitors (Vincristine)}

1) Mechanisms of drug action

Microtubules are essential proteins in cell mitosis. Although the mechanism underlying the action of microtubule inhibitors (especially vincristine sulfate) has not yet been clarified, vinca alkaloids bind directly to tubulin in microtubules and inhibit tubulin polymerization, thereby stopping cell division and resulting in cytotoxicity. Taxanes are depolymerization inhibitors that stabilize microtubules and induce cell death by causing hyperplastic microtubules.

2) Mechanisms of resistance to microtubule inhibitors ${ }^{28}$

Resistance to vinca alkaloids develops relatively quickly. The mechanisms are mutation of the binding site of tubulin and enhancement of extracellular excretion by the drug excretion pump ${ }^{29,30}$.

(1) Changes in the binding sites with tubulin: Changes in the sites where drugs bind with tubulin are usually caused by point mutations.

(2) Increased DNA repair activity: DNA repair enzyme activity is increased in damaged cancer cells, which improves their survival.

(3) Increased expression of export transporter: Increased expression of export transporter might be caused by epigenetic mechanisms. In particular, P-glycoprotein also excretes vinca alkaloids as a drug excretion pump, and increased expression of ABCC1, a drug excretion transporter, promotes intracellular drug excretion.

\section{Newly Developed Drugs}

Recently, several newly developed drugs, including small-molecule drugs (such as kinase inhibitors), antibody drugs, and immune modulatory drugs, have become available clinically and are effective for tumors resistant to conventional anticancer drugs. However, resistance to these drugs typically develops within 1 year, and other concerns have emerged ${ }^{31}$.

\section{Molecular Targeted Drugs}

1) Mechanisms of resistance to molecular targeted drugs

Although selected hematopoietic tumors can be cured by targeted drugs, many other tumors initially respond to a targeted drug but relapse develops because of resistance. The main mechanisms of targeted drug resistance include changes in target genes (gatekeeper mutations or secondary mutations, gene amplification), activation of collateral pathways, downstream activation of targets, and small-cell carcinoma transformation, epithelialmesenchymal transition (EMT), cancer stem cell traits, and other mechanisms.

(1) Mutations of gatekeeper genes ${ }^{32,33}$ : $\mathrm{Bcr}-\mathrm{Abl}$ resistance is related to T315I mutation. Epithelial growth factor receptor (EGFR)-tyrosine kinase inhibitor (TKI) resistance is related to T790M mutation. Anaplastic lymphoma kinase (ALK)-TKI resistance is related to L1196M mutation, and C-kit resistance is related to T670I substitution. Among solvent-front mutations, ALK resistance is related to $G$ $1202 \mathrm{R}$, and c-ros oncogene 1 (ROS1) is related to G2032R substitution.

(2) Increased expression of bypass pathways: Ligand attachment to EGFR activates the PI3K/Akt, MEK/ MAPK, and JAK/STAT pathways. EGFR-TKI resistance results in amplification of hepatocyte growth factor (cMet) and activation of insulin-like growth factor 1 receptor (IGF-1R), which affects the MEK/MAPK pathway. ALK resistance increases expression of the receptor of stem cell factor (c-kit).

(3) Activation of downstream signal pathways: EGFRTKI-resistant cells show further activation of downstream signal pathways such as PI3K/Akt, MEK/MAPK, and JAK/STAT and ultimately overcome suppression of downstream signals by TKI. Cells resistant to B-RAF inhibitors also exhibit increased expression of A-RAF and 
C-RAF, which leads to MAPK activation, thereby overcoming suppression of downstream signals by BRAF inhibitors.

(4) EMT: A reversible change from epithelial to mesenchymal cells that possess migration properties occurs during embryogenesis, and in wound healing and tumor metastasis. EMT leads to drug resistance. This phenomenon is caused by epigenetics ${ }^{34}$. The following processes occur during EMT.

(i) Disassembly of cell-cell contacts: Loss of epithelial markers (E-cadherin, claudin, occludin), a decrease in the number of structures of intercellular attachment (tight junction, adherence junction, desmosome), and, consequently, loss of cell polarity, lead to loss of cell-cell contacts.

(ii) Changes in the cytoskeleton (cytoskeletal modification): The cytoskeleton changes during EMT, and tumor cells gain cellular motility. Actin forms stress fibers, and intermediate filaments change from cytokeratin to vimentin.

(iii) Changes in gene expressions: Gene expressions controlled by the transcription factors SNAIL, ZEB, and the bHLH family change during EMT.

(iv) Acquisition of motility: Motility is acquired by the switch from E-cadherin to $\mathrm{N}$-cadherin, rearrangement of the cytoskeleton, and expression of matrix metalloproteinase.

\section{Immune Antibodies}

1) Mechanism of resistance to immune antibodies (antibody-based biopharmaceuticals)

The mechanisms of resistance to immune antibodies are decreased expression of antigens of tumor cells, increased DNA repair activity, and increased expression of drug export pumps.

2) Drug actions of rituximab

The molecular target drug rituximab is an antibody drug that selectively binds the CD20 surface antigen on the surface of B lymphocytes. It binds CD20, activates the human immune response to attack tumorous B lymphocytes, and is thus effective against B cell lymphomas ${ }^{35-37}$. The B-lymphocyte-damaging action of rituximab is likely mediated through precomplementary cytotoxicity (complement-dependent cytotoxicity) and antibodydependent cell-mediated cytotoxicity.

Rituximab lysed human-derived CD20-positive cells in the presence of human complement but did not lyse human-derived CD20-negative cells, which confirms that it has complement-dependent cytotoxicity for cells with the CD20 antigen. In addition, rituximab lysed human
CD20-positive cells in the presence of human effector cells but did not lyse human CD20-negative cells, thus confirming that it has antibody-dependent cell-mediated cytotoxicity for cells with the CD20 antigen.

3) Mechanisms of resistance to rituximab ${ }^{38-42}$

(1) Inhibition of conjunction between rituximab and CD20: Decreased expression of CD20, increased internalization of CD20, and abnormalities in the CD20 gene cause inhibition of the conjunction between rituximab and CD20.

(2) Abnormality of reactivity after conjunction between rituximab and CD20: Abnormal reactivity includes abnormal signal transduction, apoptosis, clustering to lipid rafts, and complement conjunction.

(3) Abnormality of reactivity of effector cells (NK cells, monocytes, macrophages): Abnormality of effector cells causes destruction of CD20-positive cells with rituximab.

4) Drug actions of trastuzumab

The drug actions of trastuzumab-a humanized monoclonal antibody against human epidermal growth factor receptor 2 (HER2, expressed in 15\% to $20 \%$ of breast cancers express)-include disturbance of the Ras-MAPK pathway, inhibition of proliferation of cancer cells, and inhibition of the PI3K-Akt-mTOR pathway and cause increased apoptosis of cancer cells and induction of antibody-dependent cellular cytotoxicity by NK cells, monocytes, and macrophages. However, some HER2positive cancers are refractory to trastuzumab, and half of patients responsive to trastuzumab developed resistance to it within 1 year.

5) Mechanisms of resistance to trastuzumab ${ }^{43}$

(1) Heterodimer formation with HER2 and the HER family: Trastuzumab inhibits homodimer formation of HER2/HER2. In resistant cancer cells, heterodimer formation is greater with HER2 and the HER family (HER1, HER3, HER4) than in sensitive cells. Heterodimers of HER exhibited survival and proliferation in tumor cells.

(2) Activation of downstream signaling pathways: Expression of the PI3K/Akt signaling pathway was increased in resistant cells.

6) Mechanism of drug action of immune checkpoint inhibitors

Cancer cells express neoantigen, which is different from antigens expressed in the host. The neoantigen is recognized by dendritic cells, and dendritic cells express the neoantigen with major histocompatibility complex (MHC) class I or II on their surface. CD4-positive T cells recognize the neoantigen combined with MHC class II on dendritic cells and are activated by costimuli with the 
conjunction of CD28 on the T cells and CD28 ligands (CD80 or CD86) on the dendritic cells. CD8-positive T cells become cytotoxic $\mathrm{T}$ cells (CTL) by recognizing neoantigen combined with MHC class I through $\mathrm{T}$ cell receptors. To avoid excessive immune reactions, cytotoxic $\mathrm{T}$ lymphocyte-associated protein-4 (CTLA-4) is expressed on CD4-positive T cells. Binding with CTLA-4 and CD28 on $\mathrm{T}$ cells inhibits $\mathrm{CD} 4$ proliferation and inflammatory cytokine expressions. CTL expresses programed cell death 1 (PD-1). When PD-1 on CTL binds programmed cell death ligand-1 (PD-L1) expressed on dendritic cells and/or tumor cells, cytokine production and cytotoxic activity by CTL are suppressed. Immune checkpoint inhibitors (anti-CTL antibody, anti-PD-1 antibody) block inhibitory actions caused by CTLA-4 and PD-1/PD-L1. Recently, Toffalori et al reported that in patients who experienced a relapse after allogenic hematopoietic cell transplantation, the increase in PD-1 expression on donorderived $\mathrm{T}$ cells paralleled the rise in minimal residual disease markers and the anticipated clinical relapse ${ }^{44}$.

7) Drug action of anti-CTLA-4 antibody

Anti-CTLA-4 antibody stimulates production of interferon- $\gamma$. Then, interferon- $\gamma$ activates interferon- $\gamma$ receptor on the cancer cells and activates the JAK/STAT pathway. Activating the STAT pathway leads to upregulation of $\mathrm{MCH}$ class I, thereby increasing targeting against activated $\mathrm{T}$ cells.

8) Drug actions of anti-PD-L1 antibody

Anti-PD-L1 antibody blocks interaction between PD-1 on T cells and PD-L1 on dendritic cells and tumor cells and restores suppression of the immune mechanism.

9) Mechanisms of resistance to anti-CTLA-4 antibody ${ }^{45}$

In anti-CTLA-4 antibody-resistant cells, the JAK/STAT pathway is inactivated by the presence of a decrease in the copy number of activation genes and an increase in the copy number of inhibitory genes.

10) Mechanism of resistance to anti-PD-L1 antibody ${ }^{46}$

Mutations of JAK1 or JAK2 cause dysfunction in JAK1 or 2, and inactivation of the STAT pathway leads to decreased expression of $\mathrm{MCH}$ class I. JAK mutation has been a major mechanism against anti-PD-L1 antibody.

\section{Conclusion}

Drug resistance remains a serious problem in cancer therapy, and epigenetic mechanisms appear to have an important role in drug resistance. To overcome resistance to drugs for pediatric cancers, further study of these epigenetic mechanisms is warranted.
Conflict of Interest: None declared.

\section{References}

1. Holleman A, Cheok MH, den Boer ML, et al. Geneexpression patterns in drug-resistant acute lymphoblastic leukemia cells and response to treatment. N Engl J Med. 2004;351:533-42.

2. Glasspool RM, Teodoridis JM, Brown R. Epigenetics as a mechanism driving polygenic clinical drug resistance. Br J Cancer. 2006;94:1087-92.

3. Eden A, Gaudet F, Waghmare A, Jaenisch R. Chromosomal instability and tumors promoted by DNA hypomethylation. Science. 2003;300:455.

4. Asano T, Nakamura K, Fujii H, et al. Altered expression of topoisomerase II $\alpha$ contributes to etoposide crossresistant K562/MX2 cell line by aberrant methylation. Brit J Cancer. 2005;92:1486-92.

5. Yamanishi M, Narazaki H, Asano T. Melatonin overcomes resistance to clofarabine in two leukemic cell lines by increased expression of deoxycytidine kinase. Exp Hematol. 2015;43:207-14.

6. Asano T, Narazaki H, Fujita A. Genome-wide DNA methylation profiling of $\mathrm{CpG}$ islands in a morpholino anthracycline derivative-resistant leukemia cell line: p38 $\alpha$ as a novel candidate for resistance [Internet]. Available from: $\mathrm{h}$ ttps://doi.org/10.1002/prp2.285

7. Wei SH, Brown R, Huang TH. Aberrant DNA methylation in ovarian cancer: is there an epigenetic predisposition to drug response? Ann N Y Acad Sci. 2003;983:24350.

8. Berger SL. Histone modifications in transcriptional regulation. Curr Opin Genet Dev. 2002;12:142-8.

9. Nowak SJ, Corces VG. Phosphorylation of histone H3: a balancing act between chromosome condensation and transcriptional activation. Trends Genet. 2004;20:214-20.

10. Tsuruo T. Mechanisms of multidrug resistance and implications for therapy. Jpn J Cancer Res. 1988;79:285-96.

11. Yamauchi T, Ueda T. A sensitive new method for clinically monitoring cytarabine concentrations at the DNA level in leukemic cells. Biochem Pharmacol. 2005;69:1795803.

12. Matsunaga S, Asano T, Tsutsuda-Asano A, Fukunaga Y. Indomethacin overcomes doxorubicin resistance by inhibiting Multi-drug resistance protein 1 (MRP1). Cancer Chemother Pharmacol. 2006;58:348-53.

13. Corrêa $S$, Binato R, Du Rocher B, et al. ABCB1 regulation through LRPPRC is influenced by the methylation status of the GC-100 box in its promoter. Epigenetics. 2014;9: 1172-83.

14. Arrigoni E, Galimberti S, Petrini M, Danesi R, Di Paolo A. ATP-binding cassette transmembrane transporters and their epigenetic control in cancer: an overview. Expert Opin Drug Metab Toxicol. 2016;12:1419-32.

15. Asano T, Tsutsuda-Asano A, Fukunaga Y. Indomethacin overcomes doxorubicin resistance by decreasing intracellular content of glutathione and its conjugates, and decreasing expression of $\gamma$-glutamylcysteine synthetase via promoter activity in doxorubicin-resistant leukemia cells. Cancer Chemother Pharmacol. 2009;64:715-21.

16. Hegi ME, Diserens AC, Gorlia T, et al. MGMT gene silencing and benefit from temozolomide in glioblastoma. N Engl J Med. 2005;352:997-1003.

17. Wiley JS, Taupin J, Jamieson GP, Snook M, Sawyer WH, Finch LR. Cytosinc arabinoside transport and metabolism in acute leukemias and $\mathrm{T}$ cell lympnoma. J Clin lnvest. 
1985;75:632-42.

18. Steuart CS, Burke PJ. Cytidine deaminase and the development of resistance to arabinosyl cytosine. Nat New Biol. 1971;233:109-10.

19. Richel DJ, Colly LP, Arkesteijn GJ, et al. Substrate-specific deoxycytidine kinase deficiency in 1-beta-Darabinofuranosylcytosine-resistant leukemic cells. Cancer Res. 1990;50:6515-9.

20. de Saint, Vincent BR, Déchamps M, Buttin G. The modulation of the thymidine triphosphate pool of Chinese hamster cells by dCMP deaminase and UDP reductase. Thymidine auxotrophy induced by CTP in dCMP deaminase-deficient line. J Biol Chem. 1980;255:162-7.

21. Wang S, Vrana JA, Bartimole TM, et al. Agents that down-regulate or inhibit protein kinase $C$ circumvent resistance to 1-beta-D-arabinofuranosylcytosine induced apoptosis in human leukemia cells that overexpress Bcl-2. Mol Pharmacol. 1997;52:1000-9.

22. Evans WE, Hon YY, Bomgaars L, et al. Preponderance of thiopurine S-methyltransferase deficiency and heterozygosity among patients intolerant to mercaptopurine or azathioprine. J Clin Oncol. 2001;19:2293-301.

23. Bleyer WA. The clinical pharmacology of methotrexate: new applications of an old drug. Cancer. 1978;41:36-51.

24. Jaffe N, Paed D, Farber S, et al. Favorable response of metastatic osteogenic sarcoma to pulse high-dose methotrexate with citrovorum rescue and radiation therapy. Cancer. 1973;31:1367-73.

25. Di Marco A, Casazza AM, Dasdia T, et al. Cytotoxic, antiviral, and antitumor activity of some derivatives of daunomycin (NSC-82151). Cancer Chemother Rep. 1973; 57:269-74.

26. Hinds $M$, Deisseroth $\mathrm{K}$, Mayes J, et al. Identification of a point mutation in the topoisomerase II gene from a human leukemia cell line containing an amsacrine-resistant form of topoisomerase II. Cancer Res. 1991;51:4729-31.

27. Jhaveri MS, Morrow CS. Methylation-mediated regulation of the glutathione S-transferase P1 gene in human breast cancer cells. Gene. 1998;210:1-7.

28. Zhang Y, Yang SH, Guo XL. New insights into Vinca alkaloids resistance mechanism and circumvention in lung cancer. Biomed Pharmacother. 2017;96:659-66.

29. Cabral FR, Brady RC, Schibler MJ. A mechanism of cellular resistance to drugs that interfere with microtubule assembly. Ann N Y Acad Sci. 1986;466:745-56.

30. Moscow JA, Cowan KH. Multidrug resistance. J Natl Cancer Inst. 1988;80:14-20.

31. Peters S, Zimmermann S. Management of resistance to first-line anaplastic lymphoma kinase tyrosine kinase inhibitor therapy. Curr Treat Options Oncol. 2018;19:37.

32. Khorashad JS, Kelley TW, Szankasi P, et al. BCR-ABL1 compound mutations in tyrosine kinase inhibitor-resistant CML: frequency and clonal relationships. Blood. 2013;121: 489-98.

33. Kodama T, Tsukaguchi $\mathrm{T}$, Yoshida M, Kondoh $\mathrm{O}$, Sakamoto H. Selective ALK inhibitor alectinib with potent antitumor activity in models of crizotinib resistance. Cancer Lett. 2014;351:215-21.

34. Träger MM, Dhayat SA. Epigenetics of epithelial-tomesenchymal transition in pancreatic carcinoma. Int J Cancer. 2017;141:24-32.
35. Prevodnik VK, Lavrenčak J, Horvat M, Novakovic BJ. The predictive significance of CD20 expression in B-cell lymphomas. Diagn Pathol. 2011;6:33.

36. Miyoshi H, Arakawa F, Sato K, et al. Comparison of CD 20 expression in B-cell lymphoma between newly diagnosed, untreated cases and those after rituximab treatment. Cancer Sci. 2012;103:1567-73.

37. Tokunaga T, Tomita A, Sugimoto $K$, et al. De novo diffuse large B-cell lymphoma with a CD 20 immunohistochemistry-positive and flow cytometrynegative phenotype: molecular mechanisms and correlation with rituximab sensitivity. Cancer Sci. 2014;105:35-43.

38. Johnson NA, Boyle M, Bashashati A, et al. Diffuse large B-cell lymphoma: reduced CD20 expression is associated with an inferior survival. Blood. 2009;113:3773-80.

39. Hiraga J, Tomita A, Sugimoto T, et al. Down-regulation of CD20 expression in B- cell lymphoma cells after treatment with rituximab-containing combination chemotherapies: its prevalence and clinical significance. Blood. 2009;113: 4885-93.

40. Suzuki Y, Yoshida T, Wang G, et al. Association of CD201 evels with clinicopathological parameters and its prognostic significance for patients with DLBCL. Ann Hematol. 2012;91:997-1005.

41. Lim SH, Beers SA, French RR, Johnson PWM, Glennie MJ, Cragg MS. Anti-CD20 monoclonal antibodies: historical and future perspectives. Haematologica. 2010;95:13543.

42. Tomita A, Hiraga J, Kiyoi H, et al. Epigenetic regulation of CD20 protein expression in a novel B-cell lymphoma cell line, RRBL1, established from a patient treated repeatedly with rituximab-containing chemotherapy. Int $\mathrm{J}$ Hematol. 2007;186:49-57.

43. Rockoski R Jr. The Erb/HER family of protein-tyrosine kinases and cancer. Pharmacol Res. 2014;79:34-74.

44. Toffalori C, Zito L, Gambacorta V, et al. Immune signature drives leukemia escape and relapse after hematopoietic cell transplantation. Nature Med. 2019;25:603-11.

45. Gao J, Shi LZ, Zhao H, et al. Loss of IFN- $\gamma$ pathway genes in tumor cells as a mechanism of resistance to antiCTLA-4 therapy. Cell. 2016;167:397-404. e9.

46. Zaretsky JM, Garcia-Diaz A, Shin DS, et al. Mutations associated with acquired resistance to PD-1 blockade in melanoma. N Engl J Med. 2016;375:819-29.

(Received, April 23, 2019)

(Accepted, May 1, 2020)

(J-STAGE Advance Publication, May 30, 2020)

Journal of Nippon Medical School has adopted the Creative Commons Attribution-NonCommercial-NoDerivatives 4.0 International License (https://creativecommons.org/licenses/by-nc-nd/4.0/) for this article. The Medical Association of Nippon Medical School remains the copyright holder of all articles. Anyone may download, reuse, copy, reprint, or distribute articles for non-profit purposes under this license, on condition that the authors of the articles are properly credited. 